\title{
Rechtsdurchsetzung bei Entscheidungen des IGH Möglichkeiten und Grenzen des Sicherheitsrates
}

\author{
Hans Sachs*
}

Abstract: This article addresses the impact of the decisions of the International Court of Justice (ICJ). After detecting the binding nature of judgments and of provisional measures of the ICJ, the possibilities and limits of the enforcement of the decisions of the ICJ by the Security Council according to Art. 94 (2) of the UN Charter are highlighted. The exertion of self-help is not excluded by this possibility, but there is a certain obligation for the states to address the Security Council in order to enforce the decision of the ICJ before they can legally exercise their right of self-help. However, the threat or use of force to implement a decision of an international court by self-help is illicit. The power of veto of the five permanent members of the Security Council is not restricted during the proceeding under Art. 94 (2) of the Charter.

Keywords: Internationaler Gerichtshof, zentrale Rechtsdurchsetzung, Selbsthilfe, einstweilige Maßnahmen, Vetorecht

$\mathrm{D}$ er vorliegende Beitrag behandelt die Wirkung und die Durchsetzungsmöglichkeiten von Entscheidungen des IGH. Wenn dabei trotz der begrenzten praktischen Relevanz besonderes Augenmerk auf die Durchsetzungsmöglichkeiten gelegt wird, so ist dies Folgendem geschuldet: Zum einen steigt die Akzeptanz und Nutzung des IGH zwar stetig, ${ }^{1}$ die ergangenen Urteile wurden jedoch insgesamt nur zu $80 \%$, bei nicht einvernehmlicher Anrufung des Gerichts sogar nur zu 50 \% befolgt. ${ }^{2}$ Dies ist für die Sachurteile angesichts der mangelnden zentralen Durchsetzungsinstanz auf internationaler Ebene zwar ein beachtlicher Wert. Er wird jedoch durch die Tendenz zum Primat des Rechts in den internationalen Beziehungen bei zunehmender Verflechtung und Institutionalisierung relativiert. Des Weiteren sind Veränderungen im Völkerrecht seit den 1960er und 1970er Jahren zu berücksichtigen, die die vornehmlich in dieser Zeit geführte Diskussion zu diesem Thema beeinflussen könnten. Letztlich bietet die anstehende umfassende Reform der Vereinten Nationen Anlass, die bestehenden Regelungen, die sich als wenig praktikabel erwiesen haben, zu überdenken und erweiterte Durchsetzungskompetenzen des Sicherheitsrates in Betracht zu ziehen.

\section{Wirkung}

Zunächst soll die Wirkung von Entscheidungen des IGH identifiziert werden. Mit Blick auf die unten zu diskutierende Durchsetzungsmöglichkeit werden hier nur Urteile und einstweilige Maßnahmen in Betracht gezogen.

* Hans Sachs, M.P.S., Rechtsreferendar, Hamburg.

1 Schneider, Patricia, Internationale Gerichtsbarkeit als Instrument friedlicher Streitbeilegung, Nomos, Baden-Baden 2003, S. 90 (Nicht zuletzt durch individuelle Absprache in Verträgen).

2 Schneider, Patricia; Thony, Kristina, Der Beitrag internationaler Gerichte zur Zivilisierung des Konfliktaustrags, 2000, http://www.ifsh.de/pdf/ publikationen/hb/hb127.pdf, vom 08.03.05, S. 15; Schneider (Fn 1), S. 243.

\subsection{Urteile}

Urteile des IGH haben bindende Wirkung. Gemäß Art. 94 I SVN verpflichten sich die Parteien eines Verfahrens vor dem IGH, die Entscheidung des Gerichts zu befolgen. Die Entscheidung entfaltet jedoch gemäß Art. 59 IGH-Statut über die Streitparteien und den Streitgegenstand hinaus keine bindende Wirkung - sie wirkt also nur inter partes. Das bedeutet, dass ein Urteil des IGH grundsätzlich keine Präjudizwirkung für weitere Entscheidungen des Gerichts entfaltet. ${ }^{3}$ Nichtsdestotrotz bezieht sich der IGH mit Blick auf die Kontinuität und Kohärenz seiner Rechtsprechung auf vorangegangene Entscheidungen. ${ }^{4}$

\subsection{Einstweilige Maßnahmen}

Ob aber auch einstweilige Maßnahmen des IGH nach Art. 41 des Statuts rechtlich oder lediglich moralisch bindend seien, war in der Literatur lange Zeit umstritten.

\subsubsection{LaGrand}

Dieser Streit könnte durch eine Entscheidung des IGH obsolet geworden sein. Im LaGrand-Fall hatte der IGH die vorliegende Frage erstmalig zu entscheiden. ${ }^{5}$

3 Mosler, Hermann; Oellers-Frahm, Karin in: Simma, Bruno, The Charter of the United Nations. A Commentary, 2. Auflage, Beck, München 2002 (im weiteren Mosler/Oellers-Frahm), Art. 94 Rn 2.

4 Mosler/Oellers-Frahm (Fn. 3), Art. 94 Rn 2.

5 LaGrand-Case (Germany vs. USA), 27.06.01, http://www.icj-cij.org/ icjwww/idocket/igus/igusframe.htm vom 07.03.05. Die Brüder Karl und Walter LaGrand wurden 1984 von einem Gericht in Arizona wegen Mordes während eines Banküberfalls zum Tode verurteilt. Die US-Behörden versäumten es, die deutschen Staatsbürger LaGrand über die Möglichkeit des konsularischen Beistands durch die Bundesrepublik Deutschland aufzuklären. Dazu waren sie nach Art. 36 des Wiener Abkommens über konsularische Beziehungen verpflichtet. Am 24.02.1999 wurde Karl LaGrand noch vor Klageerhebung vor dem IGH hingerichtet. Deutschland brachte den Fall am 03.03.1999 vor den IGH, doch Walter LaGrand wurde trotz einer einstweiligen Verfügung des IGH noch am selben Tag exekutiert. 
Er legt darin das Statut im Allgemeinen und die betreffende Norm im Speziellen teleologisch dahingehend aus, dass Art. 41 davor schützen soll, dass durch eine Beeinträchtigung des streitigen Rechts während des anhängigen Verfahrens die Funktion des Gerichts beschnitten wird, Rechte zu wahren. ${ }^{6}$ Des Weiteren erkennt das Gericht es als ein allgemeines Rechtsprinzip des Völkerrechts an, dass die Parteien eines Rechtsstreits während des anhängigen Verfahrens von allen Maßnahmen Abstand nehmen müssen, die die Durchsetzung des streitigen Rechts beeinträchtigen oder den Disput erschweren oder ausweiten könnten.?

Wie gesehen ${ }^{8}$ entfaltet das Urteil jedoch keine Bindungswirkung über den Einzelfall hinaus. Richterliche Entscheidungen werden in Art. 38 I d) IGH-Statut jedoch als »subsidiary means for the determination of the rules of law « genannt. Als solches Hilfsmittel wird die Entscheidung im LaGrand-Fall die weiteren Entscheidungen des IGH zumindest beeinflussen. Sie ist darüber hinaus eine weitere beachtliche Rechtsmeinung.

Es ließe sich ferner aus einem kontinentaleuropäischen rechtspositivistischen Rechtsverständnis heraus argumentieren, dass das Gericht mit der Auslegung von Normen nicht konstitutiv Recht schafft, was ihm nach ganz überwiegender Meinung verwehrt ist, ${ }^{9}$ sondern seine Entscheidungen rein deklaratorischer Natur sind und nur das ausführen, auf was sich die Vertragsparteien geeinigt haben. Dann wäre die Entscheidung im LaGrand-Fall aber lediglich die Bestätigung für die ohnehin durch das Statut eindeutig festgelegte Rechtslage. Dies widerspricht jedoch der Rechtsauffassung des Common Law, die die Rechtsfortbildung durch Richterrecht vorsieht. Um aber gerade eine solche konstitutive Rechtsfortbildung durch den IGH für das internationale Recht auszuschließen, wurde Art. 59 IGH-Statut vereinbart, der die Wirkung des Urteils wie gesehen auf die Parteien und den vorliegenden Gegenstand begrenzt.

Mangels Präjudizwirkung ist der Streit über die rechtliche Bindungswirkung von einstweiligen Maßnahmen daher nicht durch das LaGrand-Urteil hinfällig geworden.

\subsubsection{Diskussion}

Von den Autoren, die eine Bindungswirkung vorläufiger Maßnahmen verneinen, wird einerseits die Wortwahl der Formulierung in der englischen Fassung des Statuts ${ }^{10}$ an-

\footnotetext{
6 LaGrand-Case (Fn. 5), § 102.

7 LaGrand-Case (Fn. 5), § 103.

8 Oben, 1.1 .

9 Kammerhofer, Jörg, The Binding Nature of Provisional Measures of the International Court of Justice: The "Settlement « of the Issue in the LaGrandCase, Leiden Journal of International Law, 16 (2003), S. 67-83, S. 78.

10 Art. 41 IGH-Statut:

1. The Court shall have the power to indicate, if it considers that circumstances so require, any provisional measures which ought to be taken to preserve the respective rights of either party.

2 . Pending the final decision, notice of the measures suggested shall forthwith be given to the parties and to the Security Council.

(Herv. v. Verf.)
}

geführt, die in ihrer Schwäche eine Bindung ausschließe. ${ }^{11}$ Juristen würden eine andere Wortwahl finden, um eine rechtliche Bindung zu kreieren. ${ }^{12}$ Dieses Argument wird aber dadurch geschwächt, dass die Formulierung in der ebenfalls offiziellen französischen Fassung bei weitem nicht so weich ist. 13

Zum anderen wird auf Art. 94 II SVN verwiesen, der sich ausdrücklich nur auf Urteile beziehe. Da aber judgments und decisions Synonyme seien und einstweilige Maßnahmen definitiv keine Urteile, würden diese auch nicht unter Art. 94 I SVN fallen und seien somit von deren Bindungswirkung (ausdrücklich) ausgeschlossen. ${ }^{14}$

Für eine solche Annahme spricht, dass Art. 59 IGH-Statut ebenfalls von decisions spricht, hier aber nur auf Urteile (judgments) anwendbar ist, wie sich aus dem Kontext in Art. 56-60 des Statuts ergibt. ${ }^{15}$ Die Begriffe decision und judgment werden im IGH-Statut jedoch nicht durchgehend synonym verwendet. In Art. 16 II, 17 III, 24 III und 48 des Statuts wird der Begriff decision auch für verfahrensrechtliche Entscheidungen verwendet.

Insofern lässt sich Art. 94 II auch für die Gegenansicht anführen. Wenn man judgments und decisions nicht als Synonyme begreift, 16 lassen sich unter den Begriff decisions in Absatz 1 auch einstweilige Maßnahmen subsumieren. Dagegen lässt der Begriff judgments dies nicht zu. Die unterschiedliche Wortwahl spricht dafür, dass insofern zwischen Bindungswirkung (Art. 94 I) und Vollstreckung ein Unterschied gemacht werden sollte. ${ }^{17}$

Da die grammatikalische und die systematische Auslegung nicht zu zwingenden Ergebnissen führen, sind insofern die Argumente der teleologischen Auslegung der Vorschrift überzeugend. Danach kann der Zweck der Vorschrift, das streitige Recht bis zur endgültigen Entscheidung zu bewahren, nicht erreicht werden, ohne dass diese rechtliche Bindungskraft entfalten. Ohne diese kann letztlich sogar die Bindungskraft des Urteils selbst, die außer Frage steht, beeinträchtigt werden. ${ }^{18}$ Aus demselben Grund werden bindende einstweilige Maßnahmen zum Teil auch als allgemeiner Rechtsgrundsatz

11 Gross, Leo, Some Observations on Provisional Measures, in: Dinstein, Yôram (Hrsg.), International Law in the Time of Perplexity, Essays in Honour of Shabtai Rosenne, Nijhoff, Dordrecht 1989, S. 307; Lauterpacht, Hersch, The Development of International Law by the International Court, Stevens, London 1958, S. 254.

12 LaGrand-Case (Fn. 5), \$ 142.

13 »Ces mesures « statt »suggested measures « und »doivent être prises « statt »ought to be taken«, vgl. Kammerhofer (Fn. 9), S. 69.

14 LaGrand (Fn. 5), §§ 154 ff.

15 Schulte, Constanze, Compliance with the Judgments of the International Court of Justice, Oxford University Press, Oxford 2004, S. 32 f.

16 Dafür spricht die unterschiedliche Wortwahl in Absatz 1und 2.

17 Hambro, Edvard, The Binding Character of the Provisional Measures of Protection Indicated by the International Court of Justice, in: Schätzel, Walter; Schlochauer, Hans-Jürgen (Hrsg.), Rechtsfragen der internationalen Organisation. Festschrift für Hans Wehberg zu seinem 70. Geburtstag, Klostermann, Frankfurt 1956, 152, 168 f.; Schulte, S. 33.

18 Tanzi, Attila, Problems of the Enforcement of Decisions of the International Court of Justice and the Law of the United Nations, EJIL 6 (1995), S. 539-572, 568 f. 
des Völkerrechts gesehen: ${ }^{19}$ Art. 41 IGH-Statut sei lediglich die Kodifizierung des für den IGH vorgesehenen Verfahrens. ${ }^{20}$ Ein solcher Rechtsgrundsatz wird auch über das Prinzip der institutionellen Effektivität, wiederum abgeleitet aus dem allgemeinen Prinzip des Rechts, hergeleitet. Dieses verlange, dass einstweilige Maßnahmen bindend sein müssten, da ein Gericht sonst nicht über die notwendigen Instrumente verfüge, um seine Aufgabe, die Rechtswahrung, zu erfüllen. ${ }^{21}$

Jedenfalls ist von einer bindenden Wirkung der einstweiligen Maßnahmen des IGH auszugehen.

\section{Durchsetzung}

Im Folgenden werden die Möglichkeiten zur Durchsetzung von Entscheidungen des IGH dargestellt.

\subsection{Self-Executory Decisions}

Nicht alle Entscheidungen bedürfen der Umsetzung. Einige vollziehen sich selbst. Die augenscheinlichste Variante ist die Abweisung einer Klage. Aber auch Entscheidungen über Zulässigkeitsfragen und Beweiswürdigung sind self-executory. ${ }^{22}$ Des Weiteren sind Vollstreckungshandlungen nicht nötig bei Urteilen, die einer Partei etwas zusprechen, was (schon) in ihrem unmittelbaren Machtbereich liegt.23 Einen etwas schwierigeren Fall bilden Feststellungsurteile wie beispielsweise die Auslegung eines Vertrages. Während diese selbst wohl self-executory ist, ${ }^{24}$ kann die daraus entstehende Verpflichtung sehr wohl der Vollstreckung bedürfen. ${ }^{25}$

\subsection{Selbsthilfe}

Das klassische Völkerrecht bietet zur Durchsetzung von gerichtlichen Entscheidungen die Selbsthilfe. Selbsthilfe sind Handlungen in direkter Reaktion auf das völkerrechtswidrige Verhalten eines Staates mit dem Ziel, den rechtmäßigen Zustand wiederherzustellen oder zu wahren. ${ }^{26}$ Dabei muss die Handlung das letzte Mittel darstellen und verhältnismäßig sein. ${ }^{27}$

19 Elkind, Jerome B., Interim Protection. A Functional Approach, Nijhoff, Den Haag 1981, 163; Mani, Venkateshwara S., Interim Measures of Protection. Art 41 of the ICJ Statute and Art. 94 of the UN Charter, Indian Journal of International Law 10 (1970), S. 359, 365; ICJ, LaGrand-Case, § 103.

20 Elkind (Fn. 19), S. 163.

21 LaGrand-Case (Fn. 5), § 93

22 Oellers-Frahm, Karin, Zur Vollstreckung der Entscheidungen internationaler Gerichte im Völkerrecht, ZaöRV 36 (1976), S. 654-679, 656; Jennings, Robert, The Judicial Enforcement of International Obligations, ZaöRV 47 (1987), S. 3-16, 4.

23 Jenks, Wilfred, The Prospects of International Adjudication, Stevens \& Son, London 1964, S. 688.

24 Jenks (Fn. 23), S. 689

25 Anderer Ansicht Ollers-Frahm (Fn. 22), S. 656, die auch schon das Feststellungsurteil selbst als nicht self-executory versteht.

26 Ermacora, Felix, Selbsthilfe, in: Lexikon des Rechts: Völkerrecht, Luchterhand 1992, S. 287.

27 Ebd.

\subsubsection{Ausschluss durch Art. 94 II SVN?}

Fraglich ist jedoch, ob Selbsthilfe für Urteile des IGH neben Art. 94 II statthaft oder ob diese Möglichkeit ausgeschlossen ist. Ausgeschlossen könnte die Selbsthilfe unter drei verschiedenen Annahmen sein:

- Art. 94 II SVN stellt lediglich die Kodifizierung eines bestehenden völkergewohnheitsrechtlichen Grundsatzes dar, nach dem die Durchsetzung ergangener Urteile nur durch eine übergeordnete (unparteiische) Instanz statthaft ist. ${ }^{28}$

Ableiten ließe sich ein solcher Grundsatz aus dem allgemeinen Prinzip des Rechts in Verbindung mit dem Prinzip der souveränen Gleichheit der Staaten, die zusammen ein Primat des Rechts und nicht ein Primat der Macht vorschreiben. Jedoch besteht keine solche effektive übernationale Durchsetzungsinstanz. Daher kann kein allgemein verbindlicher Rechtsgrundsatz gesehen werden, der den Staaten den Rekurs auf Selbsthilfe bei internationalen Urteilen verbietet.

- Art. 94 II SVN stellt mit Blick auf das Gewaltverbot und das Primat des Rechts eine neu geschaffene, aber universal gültige Norm des Völkerrechts dar.

Hiergegen spricht ebenfalls das soeben Gesagte. Zudem ist Art. 94 II im Wortlaut auf Entscheidungen des IGH beschränkt. Selbsthilfe ist letztlich auch unterhalb der Schwelle zu Art. 2 IV SVN denkbar.

- Art. 94 II verbietet als lex specialis den Rekurs auf Selbsthilfe zur Durchsetzung von Urteilen des IGH.

Da mit Art. 94 II eine wesentlich schwächere Position für den Sicherheitsrat geschaffen wurde als es in der Völkerbundsatzung für den Völkerbundsrat vorgesehen war,29 ist jedoch zunächst unwahrscheinlich, dass die Hohen Vertragsparteien die Möglichkeit der Selbsthilfe neben Art. 94 II SVN ausschließen wollten. Des Weiteren ist eine Vetomöglichkeit der ständigen Mitglieder des Sicherheitsrates gegen die Umsetzung einer für sie oder ihre Protegés nachteiligen Entscheidung zumindest faktisch nicht auszuschließen. ${ }^{30}$ Dann ist aber der Rechtsschutz gegen diese Staaten nicht umfassend, was gegen eine Ablösung des Rechts auf Selbsthilfe durch Art. 94 II SVN spricht. Ferner ist dem Sicherheitsrat ein weites Entschließungs- und Entscheidungsermessen eingeräumt. ${ }^{31}$ In vielen Fällen ist daher mit einer Untätigkeit des Sicherheitsrats $\mathrm{zu}$ rechnen, insbesondere wenn man in Betracht zieht, dass er als politisches Gremium nach politischen und nicht nach rechtlichen Maßstäben entscheidet. ${ }^{32}$ Angesichts dessen ist den Mitgliedstaaten nicht zuzumuten, grundsätzlich auf Selbsthilfe zu verzichten.

28 Oellers-Frahm (Fn. 22), S. 668.

29 Mosler/Oellers-Frahm (Fn. 3), Art. 94 Rn 7; Jenks (Fn. 23), S. 692 f.

30 Dazu ausführlich unten 2.3.4.

31 Dazu ausführlich unten 2.3.3 und 2.3.5

32 Tatsächlich wurde eine Durchsetzung durch den Sicherheitsrat auch nur zweimal in Betracht gezogen, jeweils ohne Erfolg (Corfu-Channel-Fall http://www.icj-cij.org/icjwww/icases/icc/iccframe.htm und Iran-ContraFall, http://www.icj-cij.org/icjwww/icases/iNus/inusframe.htm vom 07.03.05). 


\subsubsection{Vorlagepflicht nach Art. 94 II vor Selbsthilfe?}

Es könnte allerdings eine Verpflichtung der Streitparteien bestehen, zunächst eine Durchsetzung des ergangenen Urteils durch den Sicherheitsrat nach Art. 94 II zu versuchen, bevor der Rekurs auf Selbsthilfe statthaft ist. Für eine solche Verpflichtung spricht die Pflicht zur friedlichen Streitbeilegung aus Art. 1 I und insbesondere Art. 2 III SVN. Die einem Akt der Selbsthilfe innewohnende Gefahr zur Eskalation steht im Widerspruch zur Verpflichtung der Mitgliedstaaten, ihre Streitigkeiten so beizulegen, dass der Weltfriede und die internationale Sicherheit nicht gefährdet werden (Art. 2 III SVN).

Selbsthilfe ist ferner nur dann als völkerrechtlich zulässig anzusehen, wenn sie das äußerste Mittel darstellt, das heißt, dass keine anderen völkerrechtlichen Mittel zur Verfügung stehen, den verletzten Rechtszustand wiederherzustellen oder zu wahren. ${ }^{33}$ Bei einem Urteil des IGH kommt aber ausdrücklich die Möglichkeit des Art. 94 II in Betracht. Insofern ist eine legitime Selbsthilfe nur im Falle einer Untätigkeit des Sicherheitsrates oder bei einer Situation möglich, bei der die Einschaltung des Sicherheitsrates für die Wahrung des Rechts zuviel Zeit in Anspruch nehmen würde. Der letzte Fall lässt sich mit Blick auf die durchschnittliche Verfahrensdauer von vier Jahren bis zu einem Spruch des $\mathrm{IGH}^{34}$ allerdings schwer konstruieren.

Letztlich spricht die Stellung des Sicherheitsrates im System der Vereinten Nationen als Hüter des Weltfriedens und der internationalen Sicherheit (Art. 24 I SVN) für eine solche Verpflichtung.

Es besteht daher eine grundsätzliche Verpflichtung der Staaten, bei einer Nichtbefolgung des Urteils durch die gegnerische Partei den Sicherheitsrat zur Durchsetzung des Urteils aufzufordern, bevor unilaterale Maßnahmen der Selbsthilfe statthaft sind. Bleibt der Sicherheitsrat jedoch untätig, so ist dem betreffenden Staat das legitime Recht auf Selbsthilfe unbenommen. Diese Möglichkeit bestärkt jedoch umso mehr die grundsätzliche Verpflichtung, sich zunächst um eine Entscheidung des Sicherheitsrates zu bemühen, da die Staaten keinen Verlust an Handlungsoptionen zu befürchten haben.

\subsubsection{Gewalt als Option der Selbsthilfe?}

Eine andere Frage ist jedoch, ob die Androhung oder Anwendung von Gewalt bei der Ausübung der Selbsthilfe ausgeschlossen ist.

Unter einer engen Auslegung des Gewaltverbots ${ }^{35}$ will eine Ansicht auch militärische Mittel der Selbsthilfe zulassen, da ein Vollstreckungskrieg nicht gegen die Ziele der UN-Charta

33 Ermacora (Fn. 26), S. 287

34 Schneider (Fn. 1), S. 91.

$35 » . .$. jede gegen die territoriale Unversehrtheit oder die politische Unabhängigkeit eines Staates gerichtete oder sonst mit den Zielen der Vereinten Nationen unvereinbare Androhung oder Anwendung von Gewalt.« (Art. 2 Ziffer 4 SVN) verstoße, in der wiederholt die besondere Stellung der internationalen Gerichtsbarkeit hervorgehoben wird. ${ }^{36}$ Darüber hinaus sei nach Art. 1 der Charta das oberste Ziel die Aufrechterhaltung des Friedens »nach den Grundsätzen der Gerechtigkeit und des Völkerrechts«. Nicht irgendein Frieden, sondern eben dieser dem Völkerrecht und der Gerechtigkeit entsprechende sei das in der Charta proklamierte Ziel und eine Gewaltanwendung zur Aufrechterhaltung dieser Prinzipien daher legitim. ${ }^{37}$

Jedoch ist mittlerweile allgemein anerkannt, dass das Gewaltverbot umfassend und nur mit den beiden Ausnahmen der Selbstverteidigung und der Maßnahmen nach Kapitel VII der SVN besteht. ${ }^{38}$ Der Satzteil »oder sonst mit den Zielen der Vereinten Nationen unvereinbar« bezieht sich daher nur auf alle nicht ausdrücklich in der Charta autorisierten Fälle von Gewaltanwendung. ${ }^{39}$

Zudem besteht die Aufgabe der internationalen Gerichtsbarkeit als Mittel der friedlichen Streitbeilegung gerade in der Vermeidung bewaffneter Konflikte. Angesichts der militärischen Maßnahmen innewohnenden Gefahr zur Eskalation und der herausragenden Stellung des Gewaltverbots als zentrale Norm der Charta ist die Selbsthilfe auf militärischem Wege daher ausgeschlossen. ${ }^{40}$

\subsubsection{Andere Mittel der Selbsthilfe}

Als Mittel der Selbsthilfe sind zunächst diplomatischer und wirtschaftlicher Druck denkbar. Des Weiteren kann öffentlicher Druck über eine mobilization of shame mittels internationaler Medien effektiv sein.

Wenn es um finanzielle Werte geht, ist die Beschlagnahme von Vermögen des Schuldnerstaates im Gläubigerstaat möglich. Der IGH hat einem solchem Bestreben Großbritanniens im Corfu-Channel-Fall ${ }^{41}$ keine Bedenken entgegengestellt, was auf eine allgemeine Akzeptanz eines solchen Vorgehens schließen lässt. ${ }^{42}$ Mit Blick auf die hervorgehobene Stellung des Rechts und der Gerichtsbarkeit in der UN-Charta ist es auch Drittstaaten in ihrem Hoheitsgebiet möglich, Vermögen zur Vollstreckung eines IGH-Urteils zu beschlagnahmen.

36 Art. 1, 33, 36, 92 ff.

37 Vulcan, Constantin, L'éxecution des decisions de la Cour internationale de Justice d'aprés la Charte des Nations Unies, Revue générale de droit international public (RGDIP), Bd. 51 (1947), S. 193-195; ebenso Reisman, W. Michael, The Enforcement of International Decisions, AJIL 63 (1969), S. 1-27, 12 f.; Ders., Nullity and Revision, Yale University Press, New Haven und London, 1971, S. $844 \mathrm{ff}$.

38 Dies gilt zumindest für die europäische Völkerrechtslehre. Aber auch in der US-amerikanischen Völkerrechtslehre wird die grundlegende Geltung selten bestritten, auch wenn insbesondere die Ausnahme der Selbstverteidigung weiter interpretiert wird. Dasselbe gilt für die US-Regierung: Der Irakkrieg wurde von dieser mit einem (präemptiven) Selbstverteidigungsrecht, sowie alternativ mit dem Mandat des Sicherheitsrates von 1990 gerechtfertigt, was zumindest eine theoretische Bestätigung der grundsätzlichen Geltung des Gewaltverbotes auch in der Sicht der USA beinhaltet.

39 Schachter, Oscar, The enforcement of International Judicial and Arbitral Decisions, AJIL 54 (1960,) S. 1-22 S. 15

40 U.a. Ajibola, Bola A., Compliance with Judgments of the International Court of Justice, in: Bulterman, M. K.; Knijer, M., Compliance with Judgments of International Courts, Nijhoff, Den Haag u. a. 1996, S. 19.

41 http://www.icj-cij.org/icjwww/icases/icc/iccframe.htm vom 08.03.05.

42 Oellers-Frahm (Fn. 22), S. 657. 
Zwar begrenzt Art. 59 des IGH-Statuts die Wirkung des Urteils auf die Parteien. Eine Vollstreckung durch Drittstaaten steht dem jedoch nicht entgegen, da sie nur dem Urteilsspruch (zwischen den Parteien) zu seiner Wirkung verhilft. Drittstaaten werden sich jedoch nur in sehr seltenen Fällen einer möglichen Retorsion des betroffenen Staates aussetzen wollen. Aus diesem Grund kann auch nicht von einer derartigen Verpflichtung von Drittstaaten ausgegangen werden. ${ }^{43}$ Etwas anderes gilt allerdings bei einer Entscheidung des Sicherheitsrates nach Art. 94 II. Sollte er die Beschlagnahme des Eigentums einer Partei beschließen, wäre dies für alle UN-Mitgliedstaaten nach Art. 25 SVN bindend. 44

Letztlich kommen Repressalien und Retorsionsmaßnahmen des Gläubigerstaates in Betracht.

\subsection{Art. 94 II SVN}

Die Durchsetzung von Entscheidungen des IGH ist in Art 94 II SVN geregelt.

\subsubsection{Umfang der Vorschrift: Urteile und einst- weilige Maßnahmen}

Fraglich ist, ob Art. 94 II SVN nur für Urteile des IGH oder auch für einstweilige Maßnahmen anwendbar ist.

Die Tatsache, dass einstweilige Maßnahmen ebenso wie Urteile bindend sind, ${ }^{45}$ spricht für die letztere Annahme. ${ }^{46}$ Das oben schon angeführte Argument, dass die Wirkung des Urteils bei einer unzureichenden Sicherung des streitigen Rechts in Frage gestellt ist, ${ }^{47}$ lässt sich auch hier anführen. Des Weiteren muss der IGH einstweilige Maßnahmen dem Sicherheitsrat gemäß Art. 41 II des IGH-Statuts anzeigen. Dies indiziert ebenfalls eine Anwendbarkeit von Art. 94 II SVN. ${ }^{48}$

Jedoch ist der Wortlaut des Art. 94 II ziemlich eindeutig (»judgment«). Des Weiteren spricht die unterschiedliche Wortwahl in Absatz 1 und 2 für die Beschränkung auf Urteile.

Art. 94 II ist daher nicht auf einstweilige Maßnahmen anwendbar.

\subsubsection{Res iudicata}

Es wird teilweise vertreten, der Sicherheitsrat hätte im Rahmen des Art. 94 II eine beschränkte inhaltliche Überprüfungskompetenz. ${ }^{49}$

Dann hätte er jedoch die Funktion einer Revisionsinstanz. Dies ist jedoch weder dem Art. 94 II zu entnehmen noch entspräche es dem Art. 60 des Statuts, der das Urteil als »final and

\footnotetext{
43 Oellers-Frahm (Fn. 22), S. 658.

44 Schachter (Fn. 38), S. 22.

45 Siehe oben, 1.2.

46 Mosler/Oellers-Frahm (Fn. 3), Rn 15

47 Oellers-Frahm (Fn. 22), S. 662.

48 Mosler/Oellers-Frahm (Fn. 3), Rn 15

49 Gormley, W. Paul, The Status of the Awards of International Tribunals: Possible Avoidance vs. Legal Enforcement, Howard Law Journal 10 (1964), S. $40-86,78$.
}

without appeal « bezeichnet. Darüber hinaus würde der Sicherheitsrat aufgrund politischer und nicht aufgrund rechtlicher Erwägungen entscheiden. ${ }^{50}$ Die res iudicata muss daher für den Sicherheitsrat unantastbar sein.

Dasselbe muss auch für Maßnahmen des Sicherheitsrates nach Kapitel VI und VII zur Durchsetzung eines Urteils gelten. Der IGH ist das juristische Hauptorgan der Vereinten Nationen (Art. 94 SVN), mit Blick auf Art. 60 des Statuts und den Wortlaut des Art. 94 II SVN (»...to give effect to the judgment.«) ist eine Einmischung oder Beeinträchtigung seitens des Sicherheitsrates nicht statthaft. 51

\subsubsection{Auswahlermessen und Verhältnis von Art. 94 II und Kapitel VI und VII SVN}

Bezüglich der möglichen Maßnahmen des Sicherheitsrates ist der Wortlaut des Art. 94 II SVN nicht eindeutig. Es sind »recommendations and measures « vorgesehen. Dabei ist insbesondere fraglich, in welchem Verhältnis Art. 94 II zu anderen Kompetenzen des Sicherheitsrates aus der Charta steht.

Es wird zum Teil vertreten, der Sicherheitsrat könne nur unter den Voraussetzungen der Kapitel VI und VII der Satzung die jeweils dort vorgesehenen Maßnahmen ergreifen, also nur, wenn es um eine Frage geht, die Frieden und Sicherheit betrifft. 52 Art. 94 II wäre dann nur eine verweisende Vorschrift.

Diese Annahme führt allerdings zu der absurden Situation, dass die obsiegende Partei erst eine Situation herbeiführen müsste, die geeignet ist, den Weltfrieden und die internationale Sicherheit zu gefährden, um die Voraussetzungen für ein Eingreifen des Sicherheitsrates zu schaffen. ${ }^{53}$

Des Weiteren sprechen der Wortlaut und die systematische Stellung des Art. 94 II in Kapitel XIV der Charta für ein selbstständiges Verfahren. ${ }^{54}$ Wäre eine Aktion des Sicherheitsrates nur unter den Voraussetzungen der Kapitel VI und VII möglich, wäre Art. 94 II zudem überflüssig. ${ }^{55}$ Aus den Protokollen wird ferner ersichtlich, dass die Kommission, die den Entwurf zu Art. 94 ausgearbeitet hat, eine Stärkung der Vollzugsmechanismen des IGH im Sinn hatte und nicht lediglich die Wiederholung der Kompetenzen aus Kapitel VI und VII. ${ }^{56}$ Dieser Zweck der Vorschrift ist unabhängig von einer Bedrohung der Sicherheit oder des Friedens.

Daher steht es dem Sicherheitsrat frei, grundsätzlich jegliche Maßnahmen nach Art. 94 II zu ergreifen, ohne Rekurs auf andere Artikel der Satzung.

Etwas anderes könnte aber für den Einsatz militärischer Mittel zur Vollstreckung gelten. Zwar schließt der Wortlaut

50 Schachter (Fn. 38), S. 21.

51 Schulte (Fn. 15), S. 49 f.

52 Reisman, AJIL 63 (1969), S. 15 f.; US Senat, Ausschuss für auswärtige Angelegenheiten, Hearings on the Charter of the United Nations, Juli 1945, S. 286-289.

53 Schachter (Fn. 38), S. 20; Oellers-Frahm (Fn. 22), S. 664

54 Mosler/Oellers-Frahm (Fn. 3), Rn 11; Uerpmann, Robert, Grenzen zentraler Rechtsdurchsetzung im Rahmen der Vereinten Nationen, Archiv des Völkerrechts 33 (1995), S. 107-130, S. 109.

55 Schachter (Fn. 37), S. 21; Tanzi (Fn. 18), S. 561.

56 Schulte (Fn. 15), S. 40. 
des Art. 94 II diese nicht aus. Aufgrund der überragenden Stellung des Gewaltverbots im System der UN-Charta ist aber davon auszugehen, dass die Befugnis zum Einsatz militärischer Gewalt ausdrücklich in Art. 94 II aufgeführt werden müsste, so wie in Art. 42 und 51. Aber auch der Charakter der Entscheidungen des IGH als Mittel zur friedlichen Streitbeilegung spricht gegen den Einsatz von Waffengewalt aufgrund von Art. 94 II. ${ }^{57}$ Daher sind derartige Maßnahmen nur unter den Voraussetzungen des Kapitels VII SVN, einer Bedrohung oder Bruch des Friedens oder einer Angriffshandlung durch die Nichtbeachtung des Urteils möglich. ${ }^{58}$

\subsubsection{Art. 27 III SVN-Veto}

Problematisch ist weiterhin, ob den ständigen Mitgliedern des Sicherheitsrates das in Art. 27 III SVN enthaltene Vetorecht bei einer Entscheidung im Rahmen des Art. 94 II SVN auch dann zusteht, wenn sie selbst am Verfahren vor dem IGH Streitpartei sind.

Wie oben festgestellt, bestehen die Regelungen des Art. 94 II SVN und des Kapitels VI unabhängig voneinander. Eine direkte Anwendung der Ausnahme in Art. 27 III SVN für Kapitel VI scheidet daher aus. Nichtsdestotrotz spricht gegen die Anwendbarkeit des Vetorechts das Prinzip der Effektivität der Rechtsprechung als Ausdruck des allgemeinen Prinzips des Rechts sowie die Stellung der internationalen Gerichtsbarkeit in der Satzung der Vereinten Nationen. Die Möglichkeit des Vetorechts macht Art. 94 II SVN für die ständigen Mitglieder und deren Protegés faktisch wirkungslos und beschneidet der obsiegenden Partei die Möglichkeit der effektiven Nutzung des Art. 94 II SVN. Es lässt sich argumentieren, dass ein solcher Effekt, der den Sinn und Zweck der Vorschrift konterkariert, ausdrücklich durch einen Bezug auf Art. 27 III SVN hätte bestätigt werden müssen. Dazu kommt, dass die Gerichtsbarkeit des IGH nicht obligatorisch ist, so dass die Mitgliedstaaten der VN die Auswirkungen von Art. 94 II SVN in ihre Erwägungen bei einer Erklärung nach Art. 36 II IGH-Statut oder der Ad-hoc-Unterwerfung einbeziehen können.

Angesichts des eben Dargestellten könnte eine Verpflichtung der ständigen Mitglieder des Sicherheitsrates entstehen, auf ihr Vetorecht im Rahmen des Art. 94 II SVN zu verzichten. Zumindest bei einer den Voraussetzungen des Kapitels VI vergleichbaren Situation und Maßnahmen des Sicherheitsrates in Form von recommendations könnte ferner eine analoge Anwendung des Art. 27 III SVN in Betracht kommen.

Gegen letztere Annahme spricht jedoch, dass Maßnahmen nach Kapitel VII, also solche, die wesentlich stärker in die Souveränitätsrechte des betroffenen Staates eingreifen, gerade nicht in Art. 27 III SVN als Ausnahme aufgeführt ist. Der Wortlaut des Art. 94 II umfasst aber auch solche measures, verbindlich nach Art. 25 SVN, die über die Möglichkeiten des

57 Mosler/Oellers-Frahm (Fn. 3), Rn 11.

58 Ebenso Mosler/Oellers-Frahm (Fn. 3), Rn 11, Schachter (Fn. 37), S. 21; Schulte (Fn. 15), S. 44.
Kapitels VI hinausgehen und den Maßnahmen in Kapitel VII entsprechen. Des Weiteren ist Art. 94 II nicht in Art. 27 III genannt, was bei einer beabsichtigen Ausnahme hätte geschehen können.

Im einzigen praktischen Fall hat das Veto der USA die Annahme einer vollstreckenden Resolution verhindert. ${ }^{59}$ Die Nichtannahme wurde vom Präsidenten festgestellt, ohne dass ein Widerspruch erhoben wurde. ${ }^{60}$

Angesichts dessen muss wohl davon ausgegangen werden, dass ein Vetorecht der ständigen Ratsmitglieder im Rahmen des Art. 94 II nicht ausgeschlossen ist, auch wenn ein solches zusätzlich zu den oben gebrachten Argumenten gegen den Grundsatz nemo iudex in re sua verstößt. Es muss insofern wohl davon ausgegangen werden, dass dieser Grundsatz entweder kein gewohnheitsrechtlich verbindliches allgemeines Rechtsprinzip des Völkerrechts darstellt, dass dieser durch die Satzung der Vereinten Nationen für den Sicherheitsrat ausgeschlossen wurde oder dass der Sicherheitsrat als politisches Organ nicht unter den Begriff des Gerichts fällt. Eine ähnliche Regelung fehlt schließlich auch für andere Entscheidungen des Sicherheitsrates.

Es ist letztlich zu berücksichtigen, dass ein Vetorecht im Rahmen des Art. 94 II sich nahtlos in die allgemeine (politische) Struktur der Charta einfügt, die durch die vorherrschende Machtstellung der fünf ständigen Ratsmitglieder geprägt ist. Wie wünschenswert auch immer, eine Ausnahme in Art. 94 II wäre überraschend. ${ }^{61}$

\subsubsection{Entschließungsermessen des Sicherheitsrates}

Art. 94 II SVN enthält ein weites Entschließungsermessen des Sicherheitsrates. ${ }^{62}$ Es wird nicht nur auf bestimmte Voraussetzungen verzichtet, unter denen der Sicherheitsrat tätig werden muss oder sollte, es wird sogar ausdrücklich darauf hingewiesen, dass der Sicherheitsrat nur tätig wird, soweit er es für notwendig erachtet. Dies stellt ein weiteres Hindernis für die Effektivität der Vorschrift dar.

\subsection{Andere Möglichkeiten der VN-Charta}

Der Sicherheitsrat ist zur Durchsetzung von Urteilen des IGH nicht auf Art. 94 II SVN beschränkt. Er kann ebenso Maßnahmen auf Kapitel VI und VII stützen. Auch die Generalversammlung kann sich nach Art. 10 SVN mit der Angelegenheit befassen. Für die Diskussion einer möglichen Ersatzbefugnis bei einer Lähmung des Sicherheitsrates durch ein Veto fehlt hier der Raum. Letztlich kommt auch ein Verfahren nach Art. 33, 34 in Betracht.

\footnotetext{
59 USA v. Nicaragua (Military and Paramilitary Actions in and against Nicaragua), Mosler/Oellers-Frahm (Fn. 3), Rn 13.

60 Mosler/Oellers-Frahm (Fn. 3), Rn 13.

61 Tanzi (Fn. 18), S. 542

62 "...to the Security Council, which may, if it deems necessary, make recommendations or decide upon measures to be taken to give effect to the judgment.«
} 


\section{VN-Reform}

Verschiedene Änderungen der einschlägigen Dokumente im Rahmen der umfassenden VN-Reform wären für eine Stärkung der Rechtsprechung des IGH wünschenswert.

Zum einen sollten die einstweiligen Maßnahmen de lege ferenda für rechtlich verbindlich erklärt werden. Eine Ausweitung des Art. 94 II SVN auf einstweilige Maßnahmen ist ebenfalls wünschenswert.

Des Weiteren sollte das Entschließungsermessen des Sicherheitsrates eingeschränkt werden. Vollstreckungsmaßnahmen können zwar auch die Gefahr einer Eskalation in sich tragen. Dies sowie die tatsächliche Realisierbarkeit der angeordneten Maßnahmen zu berücksichtigen bietet das Entscheidungsermessen des Sicherheitsrates aber noch genügend Raum.

Letztlich sollte dem Grundsatz nemo iudex in re sua entsprochen werden und eine Entscheidungsmöglichkeit der betroffenen Mitglieder des Sicherheitsrates zumindest im Rahmen des Art. 94 II ausgeschlossen werden.

Angesichts des politischen Charakters und der dort vorherrschenden Machtverhältnisse sollte auch darüber nachgedacht werden, ob der Sicherheitsrat überhaupt ein taugliches Vollzugsorgan für Entscheidungen des IGH sein kann. Stattdessen könnte ein eigenständiges Organ geschaffen werden. Zumindest über eine Berichtspflicht des Sicherheitsrates an das Gericht über seine Entscheidungsgründe sollte nachgedacht werden.

Über die Umsetzungsmöglichkeiten dieser Vorschläge sollte man sich jedoch insbesondere mit Blick auf die Einstellung der aktuellen US-Administration zur internationalen Gerichtsbarkeit nicht allzu großen Illusionen hingeben.

\section{Literatur}

Ajibola, Bola A., Compliance with Judgments of the International Court of Justice, in: Bulterman, M. K.; Knijer, M., Compliance with Judgments of International Courts, Nijhoff, Den Haag u. a. 1996

Elkind, Jerome B., Interim Protection. A Functional Approach, Nijhoff, Den Haag 1981

Ermacora, Felix, Selbsthilfe, in: Lexikon des Rechts: Völkerrecht, Luchterhand, Neuwied 1992, S. 187

Gormley, W. Paul, The Status of the Awards of International Tribunals: Possible Avoidance vs. Legal Enforcement, Howard Law Journal 10 (1964), S. 40-86

Gross, Leo, Some Observations on Provisional Measures, in: Dinstein, Yôram (Hrsg.), International Law in the Time of Perplexity, Essays in Honour of Shabtai Rosenne, Nijhoff, Dordrecht 1989
Hambro, Edvard, The Binding Character of the Provisional Measures of Protection Indicated by the International Court of Justice, in: Schätzel, Walter; Schlochauer, Hans-Jürgen (Hrsg.), Rechtsfragen der internationalen Organisation. Festschrift für Hans Wehberg zu seinem 70. Geburtstag, Klostermann, Frankfurt 1956

Jenks, Wilfred, The Prospects of International Adjudication, Stevens \& Son, London 1964

Jennings, Robert, The Judicial Enforcement of International Obligations, ZaöRV47 (1987), S. 3-16

Kammerhofer, Jörg, The Binding Nature of Provisional Measures of the International Court of Justice: The »Settlement « of the Issue in the LaGrand-Case, Leiden Journal of International Law, 16 (2003), S. 67-83

Lauterpacht, Hersch, The Development of International Law by the International Court, Stevens, London 1958

Mani, Venkateshwara S., Interim Measures of Protection. Art 41 of the ICJ Statute and Art. 94 of the UN Charter, Indian Journal of International Law 10 (1970)

Mosler, Hermann; Oellers-Frahm, Karin, Kommentar zu Art. 94, in: Simma, Bruno (Hrsg.), The Charter of the United Nations. A Commentary, 2. Auflage, Beck, München 2002

Oellers-Frahm, Karin, Zur Vollstreckung der Entscheidungen internationaler Gerichte im Völkerrecht, ZaöRV 36 (1976), S. 654-679

Reisman, W. Michael, Nullity and Revision, Yale University Press, New Haven und London, 1971

Reisman, W. Michael, The Enforcement of International Decisions, AJIL 63 (1969), S. 1-27

Schachter, Oscar, The Enforcement of International Judicial and Arbitral Decisions, AJIL 54 (1960,) S. 1-22

Schneider, Patricia; Thony, Kristina, Der Beitrag internationaler Gerichte zur Zivilisierung des Konfliktaustrags, 2000, http://www.ifsh.de/pdf/publikationen/hb/hb127.pdf vom 08.03.05

Schneider, Patricia, Internationale Gerichtsbarkeit als Instrument friedlicher Streitbeilegung, Nomos, Baden-Baden 2003

Schulte, Constanze, Compliance with the Judgments of the International Court of Justice, Oxford University Press, Oxford 2004

Tanzi, Attila, Problems of the Enforcement of Decisions of the International Court of Justice and the Law of the United Nations, EJIL 6 (1995), S. 539-572

Uerpmann, Robert, Grenzen zentraler Rechtsdurchsetzung im Rahmen der Vereinten Nationen, Archiv des Völkerrechts 33 (1995), S. 107-130

Vulcan, Constantin, L'éxecution des decisions de la Cour internationale de Jusitice d'aprés la Charte des Nations Unies, Revue générale de droit international public (RGDIP), Bd. 51 (1947), S. 193-195 